\title{
NATURE INDEX CHINA TABLES
}

China's leading institutions for high-quality science, ordered by weighted fractional count (WFC) for 2014. Also shown are the total number of articles, and the change in WFC from 2013. Articles are from the 68 journals that comprise the Nature Index (see 'How to use the index', S190).

\section{TOP 200 INSTITUTIONS}

\begin{tabular}{|c|c|c|c|c|c|}
\hline 2014 & INSTITUTION & WFC 2013 & WFC 2014 & AC 2014 & $\begin{array}{c}\text { CHANGE IN WFC } \\
2013-2014\end{array}$ \\
\hline 1 & Peking University (PKU) & 275.51 & 293.86 & 1,019 & $6.7 \%$ \\
\hline 2 & Nanjing University (NJU) & 196.52 & 215.08 & 518 & $9.4 \%$ \\
\hline 3 & Tsinghua University (TH) & 195.15 & 211.39 & 666 & $8.3 \%$ \\
\hline 4 & University of Science and Technology of China (USTC) & 175.78 & 193.90 & 561 & $10.3 \%$ \\
\hline 5 & Zhejiang University (ZJU) & 150.44 & 192.13 & 364 & $27.7 \%$ \\
\hline 6 & Fudan University & 129.42 & 166.21 & 356 & $28.4 \%$ \\
\hline 7 & Institute of Chemistry (ICCAS), CAS & 124.85 & 124.34 & 306 & $-0.4 \%$ \\
\hline 8 & Shanghai Institute of Organic Chemistry (SIOC), CAS & 105.62 & 114.25 & 210 & $8.2 \%$ \\
\hline 9 & Lanzhou University (LZU) & 69.72 & 110.38 & 186 & $58.3 \%$ \\
\hline 10 & Shanghai Jiao Tong University (SJTU) & 96.01 & 108.06 & 290 & $12.5 \%$ \\
\hline 11 & Jilin University (JLU) & 97.50 & 104.93 & 189 & $7.6 \%$ \\
\hline 12 & Wuhan University (WHU) & 98.90 & 96.93 & 164 & $-2.0 \%$ \\
\hline 13 & Xiamen University (XMU) & 76.02 & 95.56 & 215 & $25.7 \%$ \\
\hline 14 & Nankai University (NKU) & 113.52 & 93.43 & 230 & $-17.7 \%$ \\
\hline 15 & Sichuan University (SCU) & 76.83 & 93.36 & 177 & $21.5 \%$ \\
\hline 16 & Soochow University & 65.25 & 91.43 & 169 & $40.1 \%$ \\
\hline 17 & Sun Yat-sen University (SYSU) & 79.43 & 89.72 & 193 & $13.0 \%$ \\
\hline 18 & University of Chinese Academy of Sciences (UCAS) & 71.21 & 89.12 & 524 & $25.1 \%$ \\
\hline 19 & Institute of Physics (IOP), CAS & 77.24 & 87.88 & 267 & $13.8 \%$ \\
\hline 20 & East China Normal University (ECNU) & 65.56 & 83.17 & 148 & $26.9 \%$ \\
\hline 21 & Changchun Institute of Applied Chemistry (CIAC), CAS & 80.69 & 82.09 & 142 & $1.7 \%$ \\
\hline 22 & Hunan University (HNU) & 54.57 & 77.38 & 111 & $41.8 \%$ \\
\hline 23 & Hong Kong University of Science and Technology (HKUST) & 54.60 & 74.62 & 136 & $36.7 \%$ \\
\hline 24 & The University of Hong Kong (HKU) & 71.38 & 71.77 & 186 & $0.5 \%$ \\
\hline 25 & Dalian Institute of Chemical Physics (DICP), CAS & 61.90 & 71.75 & 139 & $15.9 \%$ \\
\hline 26 & East China University of Science and Technology (ECUST) & 56.75 & 71.27 & 130 & $25.6 \%$ \\
\hline 27 & Xi'an Jiaotong University (XJTU) & 42.98 & 67.79 & 170 & $57.7 \%$ \\
\hline 28 & Fujian Institute of Research on the Structure of Matter (FJIRSM), CAS & 59.54 & 64.96 & 124 & $9.1 \%$ \\
\hline 29 & Shandong University (SDU) & 39.18 & 63.00 & 158 & $60.8 \%$ \\
\hline 30 & Huazhong University of Science and Technology (HUST) & 43.04 & 57.39 & 154 & $33.3 \%$ \\
\hline 31 & Dalian University of Technology (DUT) & 61.42 & 52.36 & 96 & $-14.7 \%$ \\
\hline 32 & Shanghai Institutes for Biological Sciences (SIBS), CAS & 51.44 & 52.12 & 131 & $1.3 \%$ \\
\hline 33 & Southeast University (SEU) & 30.94 & 51.64 & 110 & $66.9 \%$ \\
\hline 34 & Beijing Normal University (BNU) & 39.81 & 50.82 & 144 & $27.7 \%$ \\
\hline 35 & Northeast Normal University (NENU) & 30.73 & 48.53 & 67 & $57.9 \%$ \\
\hline 36 & Tianjin University (TJU) & 33.90 & 46.23 & 151 & $36.4 \%$ \\
\hline 37 & Tongji University & 40.83 & 45.85 & 107 & $12.3 \%$ \\
\hline 38 & South China University of Technology (SCUT) & 30.74 & 45.79 & 91 & $49.0 \%$ \\
\hline 39 & Hefei Institutes of Physical Science (HIPS), CAS & 19.97 & 39.73 & 77 & $99.0 \%$ \\
\hline 40 & Institute of Semiconductors (IOS), CAS & 35.66 & 36.76 & 87 & $3.1 \%$ \\
\hline 41 & Fuzhou University (FZU) & 26.76 & 35.93 & 56 & $34.3 \%$ \\
\hline 42 & The Chinese University of Hong Kong (CUHK) & 39.39 & 35.82 & 110 & $-9.1 \%$ \\
\hline 43 & People's Liberation Army (PLA) & 42.90 & 35.33 & 132 & $-17.6 \%$ \\
\hline
\end{tabular}




\begin{tabular}{|c|c|c|c|c|c|}
\hline 2014 & INSTITUTION & WFC 2013 & WFC 2014 & AC 2014 & $\begin{array}{c}\text { CHANGE IN WFC } \\
2013-2014\end{array}$ \\
\hline 44 & Harbin Institute of Technology (HIT) & 36.22 & 33.06 & 63 & $-8.7 \%$ \\
\hline 45 & Chinese Academy of Medical Sciences \& Peking Union Medical College (CAMS \& PUMC) & 24.74 & 32.36 & 97 & $30.8 \%$ \\
\hline 46 & Technical Institute of Physics and Chemistry (TIPC), CAS & 29.11 & 32.08 & 70 & $10.2 \%$ \\
\hline 47 & City University of Hong Kong (CityU) & 36.51 & 31.97 & 78 & $-12.4 \%$ \\
\hline 48 & Shanghai University (SHU) & 16.49 & 31.87 & 72 & $93.2 \%$ \\
\hline 49 & Beijing Institute of Technology (BIT) & 20.11 & 31.35 & 65 & $55.9 \%$ \\
\hline 50 & Beijing University of Chemical Technology (BUCT) & 23.45 & 30.34 & 50 & $29.4 \%$ \\
\hline 51 & Shanghai Institute of Materia Medica (SIMM), CAS & 27.48 & 29.39 & 56 & $7.0 \%$ \\
\hline 52 & University of Science and Technology Beijing (USTB) & 25.79 & 28.85 & 53 & $11.9 \%$ \\
\hline 53 & Shanghai Institute of Ceramics, CAS (SICCAS) & 23.82 & 28.43 & 65 & $19.4 \%$ \\
\hline 54 & Institute of High Energy Physics (IHEP), CAS & 32.77 & 28.02 & 229 & $-14.5 \%$ \\
\hline 55 & Northwest University (NWU) & 12.04 & 26.26 & 40 & $118.2 \%$ \\
\hline 56 & Suzhou Institute of Nano-Tech and Nano-Bionics (SINANO), CAS & 25.40 & 25.94 & 66 & $2.1 \%$ \\
\hline 57 & Institute of Theoretical Physics (ITP), CAS & 24.03 & 24.56 & 65 & $2.2 \%$ \\
\hline 58 & Zhengzhou University (ZZU) & 13.77 & 23.58 & 53 & $71.3 \%$ \\
\hline 59 & Beihang University (BUAA) & 17.68 & 23.36 & 78 & $32.1 \%$ \\
\hline 60 & Southwest University (SWU) & 16.11 & 22.67 & 37 & $40.7 \%$ \\
\hline 61 & Central China Normal University (CCNU) & 17.28 & 22.59 & 67 & $30.7 \%$ \\
\hline 62 & National Center for Nanoscience and Technology (NCNST), CAS & 24.20 & 21.36 & 42 & $-11.7 \%$ \\
\hline 63 & Nanjing Tech University (NanjingTech) & 11.92 & 21.25 & 49 & $78.3 \%$ \\
\hline 64 & Lanzhou Institute of Chemical Physics (LICP), CAS & 17.19 & 20.61 & 47 & $19.9 \%$ \\
\hline 65 & National Astronomical Observatories (NAOC), CAS & 21.60 & 20.08 & 238 & $-7.0 \%$ \\
\hline 66 & Central South University (CSU) & 10.18 & 19.82 & 58 & $94.7 \%$ \\
\hline 67 & Shanghai Institute of Applied Physics (SINAP), CAS & 13.02 & 19.48 & 58 & $49.6 \%$ \\
\hline 68 & The Hong Kong Polytechnic University (PolyU) & 25.94 & 19.40 & 50 & $-25.2 \%$ \\
\hline 69 & Institute of Biophysics (IBP), CAS & 18.51 & 19.36 & 59 & $4.6 \%$ \\
\hline 70 & Chongqing University (CQU) & 12.90 & 19.09 & 34 & $48.1 \%$ \\
\hline 71 & Shandong Normal University (SDNU) & 12.73 & 19.06 & 24 & $49.8 \%$ \\
\hline 72 & University of Electronic Science and Technology of China (UESTC) & 11.98 & 18.42 & 49 & $53.7 \%$ \\
\hline 73 & Institute of Metal Research (IMR), CAS & 18.26 & 17.76 & 29 & $-2.7 \%$ \\
\hline 74 & Shenzhen Institutes of Advanced Technology (SIAT), CAS & 11.38 & 17.51 & 25 & $53.8 \%$ \\
\hline 75 & Kunming Institute of Botany (KIB), CAS & 9.98 & 17.43 & 29 & $74.6 \%$ \\
\hline 76 & China Agricultural University (CAU) & 14.65 & 17.20 & 44 & $17.4 \%$ \\
\hline 77 & China University of Geosciences (CUG) & 14.52 & 17.01 & 38 & $17.2 \%$ \\
\hline 78 & Nanjing Normal University (NNU) & 7.75 & 16.71 & 45 & $115.6 \%$ \\
\hline 79 & Henan Normal University (HTU) & 12.42 & 16.51 & 38 & $32.9 \%$ \\
\hline 80 & State Oceanic Administration (SOA) & 6.71 & 16.14 & 47 & $140.7 \%$ \\
\hline 81 & Wuhan University of Technology (WUT) & 8.59 & 16.05 & 29 & $86.9 \%$ \\
\hline 82 & Shanghai Institute of Technical Physics (SITP), CAS & 7.97 & 15.94 & 54 & $100.0 \%$ \\
\hline 83 & Yunnan University (YNU) & 11.39 & 15.80 & 36 & $38.6 \%$ \\
\hline 84 & South China Normal University (SCNU) & 6.87 & 15.72 & 35 & $128.7 \%$ \\
\hline 85 & Institute of Zoology (IOZ), CAS & 15.43 & 15.65 & 40 & $1.4 \%$ \\
\hline 86 & Ocean University of China (OUC) & 13.03 & 15.59 & 40 & $19.6 \%$ \\
\hline 87 & Xiangtan University (XTU) & 12.87 & 15.50 & 29 & $20.4 \%$ \\
\hline 88 & Wuhan Institute of Physics and Mathematics (WIPM), CAS & 10.35 & 15.34 & 53 & $48.3 \%$ \\
\hline 89 & Shanghai Institute of Microsystem and Information Technology (SIMIT), CAS & 17.08 & 15.21 & 30 & $-10.9 \%$ \\
\hline 90 & Nanjing Medical University (NJMU) & 7.27 & 14.91 & 58 & $105.1 \%$ \\
\hline 91 & Shanxi University (SXU) & 10.82 & 14.66 & 29 & $35.6 \%$ \\
\hline 92 & $\mathrm{BGI}$ & 15.71 & 14.66 & 63 & $-6.7 \%$ \\
\hline 93 & Huazhong Agricultural University (HZAU) & 10.67 & 14.38 & 31 & $34.8 \%$ \\
\hline 94 & Changzhou University (CZU) & 5.93 & 14.23 & 22 & $140.2 \%$ \\
\hline 95 & China University of Petroleum (UPC) & 7.15 & 14.17 & 37 & $98.2 \%$ \\
\hline 96 & Northwestern Polytechnical University (NPU) & 12.90 & 13.79 & 28 & $6.9 \%$ \\
\hline
\end{tabular}




\begin{tabular}{|c|c|c|c|c|c|}
\hline 2014 & INSTITUTION & WFC 2013 & WFC 2014 & AC 2014 & $\begin{array}{c}\text { CHANGE IN WFC } \\
2013-2014\end{array}$ \\
\hline 97 & Northeastern University (NEU) & 2.10 & 13.66 & 21 & $550.0 \%$ \\
\hline 98 & Institute of Genetics and Developmental Biology (IGDB), CAS & 10.20 & 13.43 & 38 & $31.7 \%$ \\
\hline 99 & Ningbo Institute of Materials Technology and Engineering (NIMTE), CAS & 14.94 & 13.28 & 23 & $-11.1 \%$ \\
\hline 100 & Beijing University of Technology (BJUT) & 7.67 & 13.27 & 29 & $73.0 \%$ \\
\hline 101 & Shanghai Normal University (SHNU) & 3.75 & 13.20 & 24 & $251.8 \%$ \\
\hline 102 & China Academy of Engineering Physics (CAEP) & 13.27 & 13.11 & 50 & $-1.2 \%$ \\
\hline 103 & Qingdao University of Science and Technology (QUST) & 10.66 & 13.08 & 25 & $22.7 \%$ \\
\hline 104 & Shaanxi Normal University (SNNU) & 11.80 & 12.95 & 22 & $9.8 \%$ \\
\hline 105 & Nanjing University of Aeronautics and Astronautics (NUAA) & 6.58 & 12.84 & 26 & $95.0 \%$ \\
\hline 106 & Anhui Normal University (AHNU) & 5.96 & 12.80 & 15 & $114.8 \%$ \\
\hline 107 & Hong Kong Baptist University (HKBU) & 12.77 & 12.78 & 33 & $0.1 \%$ \\
\hline 108 & South University of Science and Technology of China (SUSTC) & 2.24 & 12.52 & 31 & $458.3 \%$ \\
\hline 109 & China Meteorological Administration (CMA) & 9.93 & 12.34 & 35 & $24.3 \%$ \\
\hline 110 & Wenzhou University (WZU) & 8.05 & 11.97 & 21 & $48.6 \%$ \\
\hline 111 & Research Center for Eco-Environmental Sciences (RCEES), CAS & 6.60 & 11.92 & 20 & $80.5 \%$ \\
\hline 112 & South China Sea Institute of Oceanology (SCSIO), CAS & 10.59 & 11.82 & 23 & $11.6 \%$ \\
\hline 113 & Institute of Oceanology, CAS (IOCAS) & 5.12 & 11.48 & 22 & $124.4 \%$ \\
\hline 114 & Henan University (HENU) & 12.53 & 11.35 & 18 & $-9.4 \%$ \\
\hline 115 & Institute of Atmospheric Physics (IAP), CAS & 17.87 & 11.34 & 38 & $-36.5 \%$ \\
\hline 116 & Zhejiang University of Technology (ZJUT) & 8.03 & 11.10 & 22 & $38.2 \%$ \\
\hline 117 & China Pharmaceutical University (CPU) & 3.88 & 10.87 & 24 & $180.1 \%$ \\
\hline 118 & National University of Defense Technology (NUDT) & 16.16 & 10.68 & 44 & $-33.9 \%$ \\
\hline 119 & National Institute of Biological Sciences, Beijing (NIBS) & 11.73 & 10.65 & 31 & $-9.2 \%$ \\
\hline 120 & Jinan University (JNU) & 4.31 & 10.62 & 30 & $146.3 \%$ \\
\hline 121 & Hunan Normal University (HUNNU) & 11.12 & 10.38 & 17 & $-6.7 \%$ \\
\hline 122 & Nanchang University (NCU) & 10.06 & 9.75 & 22 & $-3.1 \%$ \\
\hline 123 & Xidian University & 3.78 & 9.65 & 12 & $155.5 \%$ \\
\hline 124 & Institute of Microbiology (IM), CAS & 9.09 & 9.59 & 24 & $5.6 \%$ \\
\hline 125 & Nanjing University of Posts and Telecommunications (NUPT) & 8.28 & 9.54 & 21 & $15.2 \%$ \\
\hline 126 & Institute of Geology and Geophysics (IGG), CAS & 7.94 & 9.48 & 22 & $19.3 \%$ \\
\hline 127 & Renmin University of China (RUC) & 8.89 & 9.46 & 24 & $6.4 \%$ \\
\hline 128 & China Earthquake Administration (CEA) & 9.50 & 9.46 & 26 & $-0.5 \%$ \\
\hline 129 & Shanghai Institute of Optics and Fine Mechanics (SIOM), CAS & 9.82 & 9.19 & 17 & $-6.4 \%$ \\
\hline 130 & Qingdao Institute of Bioenergy and Bioprocess Technology (QIBEBT), CAS & 2.90 & 9.10 & 19 & $213.9 \%$ \\
\hline 131 & Guangzhou Institutes of Biomedicine and Health (GIBH), CAS & 19.60 & 8.91 & 18 & $-54.6 \%$ \\
\hline 132 & Jiangsu Normal University (JSNU) & 5.61 & 8.66 & 13 & $54.4 \%$ \\
\hline 133 & Hebei University (HBU) & 7.86 & 8.11 & 13 & $3.1 \%$ \\
\hline 134 & Changchun Institute of Optics, Fine Mechanics and Physics (CIOMP), CAS & 10.33 & 7.95 & 16 & $-23.1 \%$ \\
\hline 135 & University of Shanghai for Science and Technology (USST) & 4.15 & 7.94 & 12 & $91.5 \%$ \\
\hline 136 & Hangzhou Normal University (HZNU) & 9.52 & 7.94 & 33 & $-16.6 \%$ \\
\hline 137 & Shenzhen University (SZU) & 5.05 & 7.73 & 26 & $53.0 \%$ \\
\hline 138 & Jiangxi Normal University (JXNU) & 3.14 & 7.71 & 23 & $146.0 \%$ \\
\hline 139 & Hefei University of Technology (HFUT) & 8.97 & 7.70 & 16 & $-14.1 \%$ \\
\hline 140 & Nanjing University of Science and Technology (NUST) & 6.43 & 7.60 & 18 & $18.3 \%$ \\
\hline 141 & Yangzhou University (YZU) & 4.67 & 7.47 & 13 & $60.1 \%$ \\
\hline 142 & Anhui University (AHU) & 3.71 & 7.28 & 18 & $96.1 \%$ \\
\hline 143 & Zhejiang Normal University (ZJNU) & 8.44 & 7.27 & 15 & $-13.9 \%$ \\
\hline 144 & Northwest A\&F University (NWAFU) & 9.90 & 7.26 & 19 & $-26.6 \%$ \\
\hline 145 & Shantou University (STU) & 7.13 & 6.99 & 18 & $-1.9 \%$ \\
\hline 146 & Institute of Botany (IBCAS) & 3.02 & 6.71 & 15 & $122.3 \%$ \\
\hline 147 & Southern Medical University (SMU) & 3.47 & 6.70 & 23 & $93.4 \%$ \\
\hline 148 & Chinese Academy of Agricultural Sciences (CAAS) & 8.10 & 6.66 & 26 & $-17.8 \%$ \\
\hline 149 & Hebei Normal University & 1.40 & 6.59 & 15 & $371.3 \%$ \\
\hline
\end{tabular}




\begin{tabular}{|c|c|c|c|c|c|}
\hline 2014 & INSTITUTION & WFC 2013 & WFC 2014 & AC 2014 & $\begin{array}{c}\text { CHANGE IN WFC } \\
2013-2014\end{array}$ \\
\hline 150 & Purple Mountain Observatory (PMO), CAS & 6.31 & 6.40 & 97 & $1.5 \%$ \\
\hline 151 & Heilongjiang University (HLJU) & 6.67 & 6.39 & 10 & $-4.2 \%$ \\
\hline 152 & North China Electric Power University (NCEPU) & 6.50 & 6.27 & 14 & $-3.6 \%$ \\
\hline 153 & Jiangnan University (JU) & 8.91 & 6.21 & 12 & $-30.3 \%$ \\
\hline 154 & Nanjing University of Information Science and Technology (NUIST) & 7.74 & 6.09 & 25 & $-21.3 \%$ \\
\hline 155 & Jiangsu University (JU) & 3.42 & 6.04 & 17 & $76.4 \%$ \\
\hline 156 & Beijing Jiaotong University (BJTU) & 4.15 & 5.97 & 15 & $43.9 \%$ \\
\hline 157 & Nanjing Agricultural University (NAU) & 2.59 & 5.83 & 18 & $125.0 \%$ \\
\hline 158 & Northwest Normal University (NWNU) & 0.25 & 5.71 & 11 & $2,184.8 \%$ \\
\hline 159 & Xinjiang Technical Institute of Physics and Chemistry (XTIPC), CAS & 4.98 & 5.66 & 10 & $13.8 \%$ \\
\hline 160 & University of Jinan (UJN) & 5.57 & 5.65 & 10 & $1.4 \%$ \\
\hline 161 & Capital Medical University (CMU) & 3.05 & 5.54 & 38 & $81.6 \%$ \\
\hline 162 & Shanghai Astronomical Observatory (SHAO), CAS & 4.76 & 5.51 & 102 & $15.8 \%$ \\
\hline 163 & Xuzhou Medical University (XZMC) & 0.31 & 5.39 & 14 & $1,665.9 \%$ \\
\hline 164 & Guangxi Normal University (GXNU) & 4.60 & 5.30 & 13 & $15.3 \%$ \\
\hline 165 & Tianjin Medical University (TMC) & 6.73 & 5.21 & 17 & $-22.7 \%$ \\
\hline 166 & Yantai Institute of Coastal Zone Research (YIC), CAS & 2.14 & 4.95 & 8 & $131.2 \%$ \\
\hline 167 & Capital Normal University (CNU) & 6.65 & 4.88 & 19 & $-26.6 \%$ \\
\hline 168 & Institute of Tibetan Plateau Research (ITP), CAS & 7.22 & 4.73 & 16 & $-34.5 \%$ \\
\hline 169 & Tianjin Normal University (TJNU) & 0.94 & 4.71 & 18 & $402.1 \%$ \\
\hline 170 & Qufu Normal University (QFNU) & 3.63 & 4.67 & 9 & $28.8 \%$ \\
\hline 171 & Harbin Engineering University (HEU) & 2.52 & 4.67 & 7 & $85.1 \%$ \\
\hline 172 & Zhejiang Sci-Tech University (ZSTU) & 4.94 & 4.64 & 13 & $-6.2 \%$ \\
\hline 173 & Institute of Microelectronics (IME), CAS & 6.80 & 4.44 & 11 & $-34.7 \%$ \\
\hline 174 & Chengdu Institute of Biology (CIB), CAS & 5.45 & 4.44 & 14 & $-18.6 \%$ \\
\hline 175 & Beijing University of Posts and Telecommunications (BUPT) & 3.42 & 4.37 & 7 & $28.0 \%$ \\
\hline 176 & $\mathrm{BGl}$ & 3.82 & 4.35 & 21 & $14.0 \%$ \\
\hline 177 & Linyi University (LYU) & 3.19 & 4.29 & 18 & $34.4 \%$ \\
\hline 178 & Guizhou University (GZU) & 3.18 & 4.29 & 10 & $35.0 \%$ \\
\hline 179 & Ningbo University (NBU) & 7.56 & 4.27 & 14 & $-43.5 \%$ \\
\hline 180 & Qingdao University (QU) & 2.89 & 4.27 & 14 & $47.6 \%$ \\
\hline 181 & Institute of Vertebrate Paleontology and Paleoanthropology (IVPP), CAS & 4.19 & 4.27 & 20 & $1.8 \%$ \\
\hline 182 & Hebei University of Technology (HEBUT) & 1.65 & 4.25 & 7 & $158.0 \%$ \\
\hline 183 & Institute of Process Engineering (IPE), CAS & 3.57 & 4.22 & 14 & $18.1 \%$ \\
\hline 184 & Huaibei Normal University (HUN) & 4.78 & 4.19 & 9 & $-12.2 \%$ \\
\hline 185 & Yanshan University (YSU) & 4.03 & 4.14 & 7 & $2.8 \%$ \\
\hline 186 & Yantai University & 0.64 & 4.12 & 9 & $541.1 \%$ \\
\hline 187 & Institute of Mechanics (IM), CAS & 3.84 & 4.09 & 9 & $6.7 \%$ \\
\hline 188 & Tianjin University of Technology (TUT) & 5.53 & 4.00 & 7 & $-27.7 \%$ \\
\hline 189 & Chongqing Medical University (CQMU) & 4.31 & 3.83 & 14 & $-11.2 \%$ \\
\hline 190 & Wenzhou Medical University (WMU) & 1.49 & 3.81 & 11 & $155.7 \%$ \\
\hline 191 & Kunming University of Science and Technology (KUST) & 3.04 & 3.77 & 9 & $24.1 \%$ \\
\hline 192 & Chinese Center for Disease Control and Prevention (China CDC) & 3.20 & 3.77 & 24 & $17.7 \%$ \\
\hline 193 & Chengdu Institute of Organic Chemistry (CIOC), CAS & 4.16 & 3.76 & 8 & $-9.6 \%$ \\
\hline 194 & Donghua University (DHU) & 6.34 & 3.72 & 11 & $-41.4 \%$ \\
\hline 195 & Henan Polytechnic University (HPU) & 0.68 & 3.71 & 13 & $442.5 \%$ \\
\hline 196 & Anhui Medical University (AHMU) & 2.12 & 3.71 & 16 & $74.7 \%$ \\
\hline 197 & Hubei University (HUBU) & 3.63 & 3.68 & 7 & $1.4 \%$ \\
\hline 198 & National Space Science Center (NSSC), CAS & 2.87 & 3.65 & 28 & $27.3 \%$ \\
\hline 199 & Institute of Coal Chemistry (ICC), CAS & 1.69 & 3.61 & 9 & $113.8 \%$ \\
\hline 200 & Institute of Electrical Engineering (IEE), CAS & 2.09 & 3.60 & 7 & $72.3 \%$ \\
\hline
\end{tabular}




\section{TOP 50 INSTITUTIONS IN LIFE SCIENCES}

\begin{tabular}{|c|c|c|c|c|c|}
\hline 2014 & INSTITUTION & WFC 2013 & WFC 2014 & AC 2014 & $\begin{array}{c}\text { CHANGE IN WFC } \\
2013-2014\end{array}$ \\
\hline 1 & Shanghai Institutes for Biological Sciences (SIBS), CAS & 49.30 & 51.12 & 125 & $3.7 \%$ \\
\hline 2 & Peking University (PKU) & 41.54 & 47.77 & 156 & $15.0 \%$ \\
\hline 4 & Shanghai Jiao Tong University (SJTU) & 20.50 & 27.31 & 92 & $33.2 \%$ \\
\hline 5 & Zhejiang University (ZJU) & 16.53 & 23.03 & 75 & $39.4 \%$ \\
\hline 7 & Sun Yat-sen University (SYSU) & 11.21 & 21.47 & 60 & $91.5 \%$ \\
\hline 8 & Chinese Academy of Medical Sciences \& Peking Union Medical College (CAMS \& PUMC) & 13.44 & 19.02 & 70 & $41.5 \%$ \\
\hline 9 & Shandong University (SDU) & 6.63 & 18.28 & 40 & $175.7 \%$ \\
\hline 10 & Fudan University & 21.72 & 17.72 & 89 & $-18.4 \%$ \\
\hline 11 & University of Science and Technology of China (USTC) & 19.07 & 17.52 & 45 & $-8.1 \%$ \\
\hline 15 & $\mathrm{BGI}$ & 15.56 & 13.75 & 62 & $-11.7 \%$ \\
\hline 16 & Institute of Genetics and Developmental Biology (IGDB), CAS & 10.18 & 13.31 & 37 & $30.8 \%$ \\
\hline 17 & Wuhan University (WHU) & 15.08 & 12.91 & 29 & $-14.4 \%$ \\
\hline 18 & The University of Hong Kong (HKU) & 10.28 & 12.49 & 46 & $21.5 \%$ \\
\hline 19 & Nanjing University (NJU) & 9.32 & 11.40 & 35 & $22.3 \%$ \\
\hline 20 & Huazhong University of Science and Technology (HUST) & 8.71 & 10.59 & 42 & $21.6 \%$ \\
\hline 21 & Huazhong Agricultural University (HZAU) & 7.61 & 10.03 & 23 & $31.9 \%$ \\
\hline 22 & Hong Kong University of Science and Technology (HKUST) & 5.48 & 9.85 & 18 & $79.7 \%$ \\
\hline 23 & National Institute of Biological Sciences, Beijing (NIBS) & 9.77 & 9.38 & 26 & $-4.0 \%$ \\
\hline 24 & Xiamen University (XMU) & 5.83 & 9.16 & 31 & $57.1 \%$ \\
\hline 31 & The Chinese University of Hong Kong (CUHK) & 6.44 & 7.70 & 33 & $19.5 \%$ \\
\hline 32 & Sichuan University (SCU) & 2.15 & 7.13 & 38 & $231.4 \%$ \\
\hline 33 & Tongji University & 8.01 & 6.70 & 36 & $-16.4 \%$ \\
\hline 34 & Soochow University & 5.12 & 6.35 & 22 & $24.1 \%$ \\
\hline 35 & Institute of Botany (IBCAS) & 2.97 & 5.68 & 13 & $90.8 \%$ \\
\hline 36 & Tianjin Medical University (TMC) & 5.79 & 5.21 & 17 & $-10.1 \%$ \\
\hline 37 & Shanghai Institute of Materia Medica (SIMM), CAS & 10.26 & 4.99 & 18 & $-51.3 \%$ \\
\hline 38 & Central South University (CSU) & 1.72 & 4.11 & 21 & $138.5 \%$ \\
\hline 39 & Beijing Institute of Genomics (BIG), CAS & 3.61 & 4.03 & 19 & $11.9 \%$ \\
\hline 40 & Capital Medical University (CMU) & 2.93 & 4.02 & 32 & $37.5 \%$ \\
\hline 41 & Institute of Vertebrate Paleontology and Paleoanthropology (IVPP), CAS & 3.96 & 3.95 & 18 & $-0.2 \%$ \\
\hline 42 & Southeast University (SEU) & 2.51 & 3.91 & 11 & $55.9 \%$ \\
\hline 43 & Chinese Academy of Agricultural Sciences (CAAS) & 6.53 & 3.63 & 17 & $-44.4 \%$ \\
\hline 44 & Wenzhou Medical University (WMU) & 1.08 & 3.49 & 10 & $224.7 \%$ \\
\hline 45 & Southern Medical University (SMU) & 2.77 & 3.48 & 14 & $26.0 \%$ \\
\hline 46 & Anhui Medical University (AHMU) & 2.12 & 3.41 & 14 & $61.0 \%$ \\
\hline 47 & Yunnan University (YNU) & 1.11 & 3.30 & 10 & $196.1 \%$ \\
\hline 48 & Xi'an Jiaotong University (XJTU) & 0.81 & 3.27 & 16 & $303.9 \%$ \\
\hline 49 & Kunming Institute of Zoology (KIZ), CAS & 3.19 & 3.21 & 16 & $0.6 \%$ \\
\hline
\end{tabular}




\section{TOP 50 INSTITUTIONS IN CHEMISTRY}

\begin{tabular}{|c|c|c|c|c|c|}
\hline 2014 & INSTITUTION & WFC 2013 & WFC 2014 & AC 2014 & $\begin{array}{l}\text { CHANGE IN WFC } \\
2013-2014\end{array}$ \\
\hline 1 & Peking University (PKU) & 142.60 & 152.25 & 378 & $6.8 \%$ \\
\hline 2 & Nanjing University (NJU) & 117.69 & 129.85 & 229 & $10.3 \%$ \\
\hline 3 & Zhejiang University (ZJU) & 86.44 & 129.11 & 189 & $49.4 \%$ \\
\hline 4 & Institute of Chemistry (ICCAS), CAS & 119.81 & 120.18 & 287 & $0.3 \%$ \\
\hline 5 & Fudan University & 80.30 & 117.15 & 198 & $45.9 \%$ \\
\hline 6 & Shanghai Institute of Organic Chemistry (SIOC), CAS & 105.23 & 113.47 & 206 & $7.8 \%$ \\
\hline 7 & University of Science and Technology of China (USTC) & 93.78 & 111.24 & 233 & $18.6 \%$ \\
\hline 8 & Tsinghua University (TH) & 92.19 & 107.43 & 249 & $16.5 \%$ \\
\hline 9 & Lanzhou University (LZU) & 50.35 & 88.25 & 124 & $75.3 \%$ \\
\hline 10 & Jilin University (JLU) & 73.64 & 80.64 & 133 & $9.5 \%$ \\
\hline 11 & Changchun Institute of Applied Chemistry (CIAC), CAS & 79.52 & 79.25 & 134 & $-0.3 \%$ \\
\hline 12 & Sichuan University (SCU) & 68.45 & 75.15 & 107 & $9.8 \%$ \\
\hline 13 & Xiamen University (XMU) & 59.87 & 73.56 & 146 & $22.9 \%$ \\
\hline 14 & Dalian Institute of Chemical Physics (DICP), CAS & 61.65 & 70.08 & 135 & $13.7 \%$ \\
\hline 15 & Hunan University (HNU) & 51.90 & 69.09 & 91 & $33.1 \%$ \\
\hline 16 & Nankai University (NKU) & 86.00 & 67.62 & 166 & $-21.4 \%$ \\
\hline 17 & East China University of Science and Technology (ECUST) & 53.04 & 66.33 & 112 & $25.0 \%$ \\
\hline 18 & Wuhan University (WHU) & 63.32 & 65.98 & 100 & $4.2 \%$ \\
\hline 19 & Fujian Institute of Research on the Structure of Matter (FJIRSM), CAS & 55.22 & 63.42 & 121 & $14.9 \%$ \\
\hline 20 & Soochow University & 34.69 & 62.89 & 104 & $81.3 \%$ \\
\hline 21 & Sun Yat-sen University (SYSU) & 52.10 & 56.43 & 97 & $8.3 \%$ \\
\hline 22 & University of Chinese Academy of Sciences (UCAS) & 47.89 & 55.93 & 278 & $16.8 \%$ \\
\hline 23 & East China Normal University (ECNU) & 41.54 & 55.26 & 86 & $33.0 \%$ \\
\hline 24 & Shanghai Jiao Tong University (SJTU) & 45.14 & 51.63 & 93 & $14.4 \%$ \\
\hline 25 & Northeast Normal University (NENU) & 25.65 & 42.59 & 54 & $66.0 \%$ \\
\hline 26 & Dalian University of Technology (DUT) & 47.16 & 41.33 & 76 & $-12.4 \%$ \\
\hline 27 & South China University of Technology (SCUT) & 28.75 & 39.39 & 73 & $37.0 \%$ \\
\hline 28 & Tianjin University (TJU) & 25.59 & 37.21 & 131 & $45.4 \%$ \\
\hline 29 & The University of Hong Kong (HKU) & 39.04 & 36.31 & 60 & $-7.0 \%$ \\
\hline 30 & Hong Kong University of Science and Technology (HKUST) & 25.86 & 36.24 & 72 & $40.2 \%$ \\
\hline 31 & Shandong University (SDU) & 19.69 & 31.88 & 57 & $61.9 \%$ \\
\hline 32 & Fuzhou University (FZU) & 26.51 & 31.23 & 49 & $17.8 \%$ \\
\hline 33 & Southeast University (SEU) & 9.78 & 28.73 & 57 & $193.7 \%$ \\
\hline 34 & Technical Institute of Physics and Chemistry (TIPC), CAS & 22.50 & 28.42 & 61 & $26.3 \%$ \\
\hline 35 & Beijing University of Chemical Technology (BUCT) & 20.35 & 28.33 & 46 & $39.2 \%$ \\
\hline 36 & Tongji University & 19.51 & 28.04 & 44 & $43.7 \%$ \\
\hline 37 & Huazhong University of Science and Technology (HUST) & 12.58 & 26.68 & 58 & $112.0 \%$ \\
\hline 38 & Xi'an Jiaotong University (XJTU) & 10.72 & 26.50 & 67 & $147.1 \%$ \\
\hline 39 & Shanghai Institute of Materia Medica (SIMM), CAS & 18.28 & 24.56 & 39 & $34.4 \%$ \\
\hline 40 & Northwest University (NWU) & 10.29 & 21.95 & 25 & $113.3 \%$ \\
\hline 41 & Shanghai University (SHU) & 8.57 & 21.54 & 43 & $151.1 \%$ \\
\hline 42 & Beijing Normal University (BNU) & 10.56 & 21.11 & 37 & $99.9 \%$ \\
\hline 43 & Hefei Institutes of Physical Science (HIPS), CAS & 8.68 & 20.92 & 40 & $141.1 \%$ \\
\hline 44 & Southwest University (SWU) & 12.51 & 20.67 & 26 & $65.2 \%$ \\
\hline 45 & Beijing Institute of Technology (BIT) & 16.95 & 20.47 & 42 & $20.7 \%$ \\
\hline 46 & Institute of Physics (IOP), CAS & 14.31 & 20.41 & 75 & $42.7 \%$ \\
\hline 47 & Nanjing Tech University (NanjingTech) & 11.04 & 19.81 & 42 & $79.4 \%$ \\
\hline 48 & National Center for Nanoscience and Technology (NCNST), CAS & 21.82 & 19.69 & 38 & $-9.8 \%$ \\
\hline 49 & Harbin Institute of Technology (HIT) & 14.15 & 19.20 & 35 & $35.7 \%$ \\
\hline 50 & Lanzhou Institute of Chemical Physics (LICP), CAS & 15.25 & 18.86 & 44 & $23.7 \%$ \\
\hline
\end{tabular}




\section{TOP 50 INSTITUTIONS IN PHYSICAL SCIENCES}

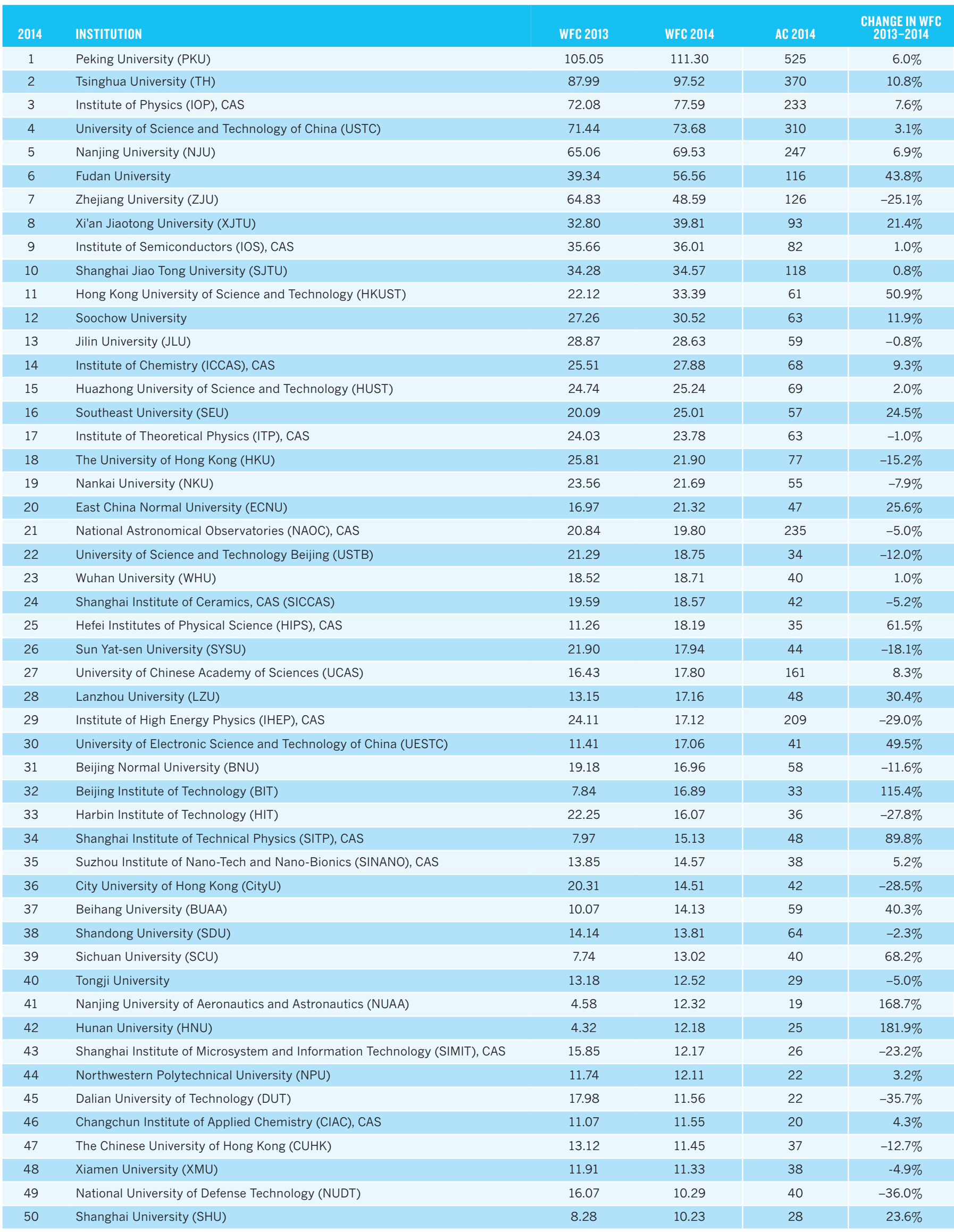




\section{TOP 25 INSTITUTIONS IN EARTH AND ENVIRONMENTAL SCIENCES}

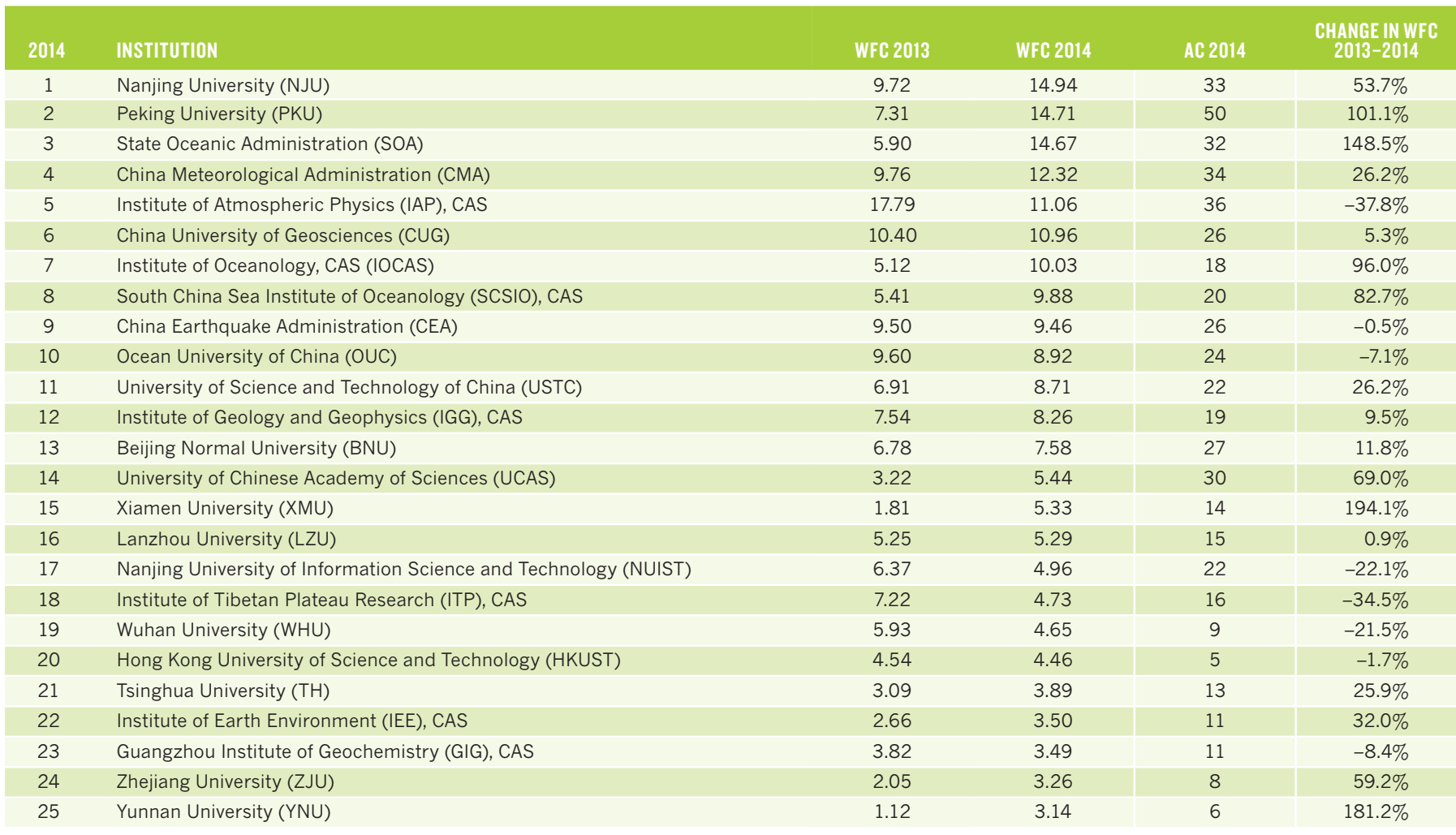

\section{TOP 25 INSTITUTIONS IN NATURE AND SCIENCE}

\begin{tabular}{|c|c|c|c|c|c|}
\hline 2014 & INSTITUTION & WFC 2013 & WFC 2014 & AC 2014 & $\begin{array}{l}\text { CHANGE IN WFC } \\
2013-2014\end{array}$ \\
\hline 1 & Peking University (PKU) & 4.10 & 6.48 & 28 & $58.0 \%$ \\
\hline 2 & Tsinghua University (TH) & 5.43 & 4.90 & 20 & $-9.8 \%$ \\
\hline 3 & $\mathrm{BGl}$ & 1.78 & 2.84 & 14 & $59.1 \%$ \\
\hline 4 & Shanghai Institutes for Biological Sciences (SIBS), CAS & 5.44 & 2.70 & 10 & $-50.4 \%$ \\
\hline 5 & Institute of Biophysics (IBP), CAS & 0.13 & 2.56 & 7 & $1,856.6 \%$ \\
\hline 6 & Zhejiang University (ZJU) & 1.70 & 2.19 & 8 & $28.9 \%$ \\
\hline 7 & National Institute of Biological Sciences, Beijing (NIBS) & 1.12 & 2.11 & 4 & $87.3 \%$ \\
\hline 8 & Dalian Institute of Chemical Physics (DICP), CAS & 0.89 & 1.56 & 4 & $75.7 \%$ \\
\hline 9 & University of Chinese Academy of Sciences (UCAS) & 0.50 & 1.20 & 7 & $138.5 \%$ \\
\hline 10 & Ocean University of China (OUC) & 0.17 & 1.17 & 3 & $600.0 \%$ \\
\hline 11 & Institute of Physics (IOP), CAS & 0.95 & 1.12 & 5 & $18.0 \%$ \\
\hline 12 & Huazhong University of Science \& Technology (HUST) & 0.05 & 0.92 & 1 & $1,733.3 \%$ \\
\hline 13 & Shanghai Institute of Organic Chemistry (SIOC), CAS & & 0.92 & 1 & \\
\hline 14 & Yanshan University & 0.73 & 0.82 & 1 & $11.6 \%$ \\
\hline 15 & University of Science and Technology of China (USTC) & 1.69 & 0.82 & 7 & $-51.6 \%$ \\
\hline 16 & Harbin Institute of Technology (HIT) & 0.07 & 0.80 & 1 & $1,020.0 \%$ \\
\hline 17 & China National Genebank (CNGB) & & 0.64 & 7 & \\
\hline 18 & Second Military Medical University (SMMU) & & 0.64 & 3 & \\
\hline 19 & Nanjing University (NJU) & 0.05 & 0.58 & 2 & $1,008.3 \%$ \\
\hline 20 & Chinese Academy of Agricultural Sciences (CAAS) & 2.84 & 0.56 & 5 & $-80.5 \%$ \\
\hline 21 & Shanghai Jiao Tong University (SJTU) & 0.18 & 0.54 & 1 & $193.1 \%$ \\
\hline 22 & Nanjing Institute of Geology and Palaeontology, CAS & 0.75 & 0.53 & 2 & $-29.5 \%$ \\
\hline 23 & Chinese Academy of Medical Sciences \& Peking Union Medical College (CAMS \& PUMC) & 0.13 & 0.53 & 3 & $322.5 \%$ \\
\hline 24 & Yunnan University & 0.80 & 0.50 & 1 & $-37.5 \%$ \\
\hline 25 & Institute of Oceanology, CAS (IOCAS) & & 0.50 & 1 & \\
\hline
\end{tabular}

Weighted fractional count (WFC) for each institution is shown to two decimal places only. When two or more institutions have the same WFC, their positions are determined by the thousandth place (or beyond).
These results are based on the most recent data available as of 14 September 2015. Owing to continual refinements of the data, the figures in the database are liable to change and might differ to those printed in the supplements. 